\title{
In vivo and in vitro assessment and proteomic analysis of the ef- fectiveness of physical treatments in reducing allergenicity of hazelnut proteins
}

\author{
Elisabetta De Angelis ${ }^{1}$, Danilo Di Bona ${ }^{2}$, Rosa Pilolli' ${ }^{1}$, Roberto Loiodice ${ }^{2}$, Anna Luparelli ${ }^{1}$, Lucia Giliberti $^{2}$, Maria \\ Pia Rossi ${ }^{2}$, Luigi Macchia ${ }^{2}$ and Linda Monaci ${ }^{*}$ \\ 1 Institute of Science of Food Production - National Research Council of Italy (ISPA-CNR) Via Amendola \\ 122/O, 70126 Bari, Italy; elisabetta.deangelis@ispa.cnr.it, rosa.pilolli@ispa.cnr.it, linda.monaci@ispa.cnr.it, \\ anna.luparelli@uniba.it \\ 2 Department of Emergency and Organ Transplantation (DETO), School and Chair of Allergology and Clini- \\ cal Immunology, University of Bari -Aldo Moro, Bari, Italy Danilo.dibona@uniba.it, Robertoloiodice@tis- \\ cali.it, Lucia.giliberti@uniba.it, Mariapia.rossi@uniba.it, Luigi.macchia@uniba.it, \\ * Correspondence: linda.monaci@ispa.cnr.it
}

\begin{abstract}
Hazelnut is a widespread nut species, especially in Europe, that can be consumed raw or roasted, due to its pleasant taste and nutritional properties. Despite the renowned beneficial properties, hazelnuts contain several proteins capable of inducing food allergy in sensitized individuals such as Cor a 2 (profilin), Cor a 8 (lipid transfer protein), Cor a 9 (11S seed storage globulin, leguminlike) and Cor a 11 ( $7 \mathrm{~S}$ seed storage globulin, vicilin-like). In the present paper we investigated the effectiveness of autoclave based treatments in decreasing the allergic potential of hazelnut as also assessed by submitting the treated material to in vivo skin prick test and to in vitro immunoblot analysis with sera of allergic individuals exposed to the treated food material. This preliminary analysis shows that autoclave treatment, preceded by hydration and/or followed by drying, seems to be a promising approach and appears to be effective in reducing the allergenicity of hazelnut in most patients, probably due to the denaturation of most major and minor allergenic proteins. This work will open to the opportunity to produce, in perspective, hypoallergenic hazelnut derivatives tolerated by the allergic subjects.
\end{abstract}

Keywords: food allergy, allergens, allergenicity reduction, skin prick test, proteomic analysis, physical treatments

\section{Introduction}

Hazelnut is one of the foods most frequently responsible for allergic reactions with an estimated prevalence in Europe of approximately $9.3 \%$ of hazelnut specific IgE detected in adults (20 - 54 years)[1]. Several allergens have been identified in hazelnuts and included in the WHO-IUIS list of allergens [2], but the most studied proteins in relation to food allergy are Cor a 2 (profilin), Cor a 8 (lipid transfer protein), Cor a 9 (11S seed storage globulin, legumin-like) and Cor a 11 (7S seed storage globulin, vicilin-like). Sensitization to Cor a 2 (thermo- and gastro-labile) is associated to oral allergic syndrome, whereas sensitization to Cor a 8, 9 or 11 (thermo- and gastro-resistant) is generally associated to systemic reactions, even severe and life-threatening. Hazelnut consumption is widespread all over the world, especially in Europe and in the Mediterranean diet, due to its pleasant taste and nutritional properties. It can be consumed fresh or roasted, but it is mostly used as ingredient in several food products or preparations (spreads, bakery, pastry, chocolates, and confectionary products). Although some common preparation processes can modify food proteins, they do not necessarily reduce the allergenic potential of the containing food, especially in case of thermo-resistant proteins; this is just the case of nut proteins belonging to the class of seed storage globulins, which maintain their allergenicity even after roasting. The European regulation 1169/2011 on the labeling of food products mandates the obligatory and clear labeling for 14 allergenic ingredients, including 
nuts [3]. On the other hand, hazelnut might contaminate food products as "hidden allergen", as a result of cross-contamination occurring during manufacturing and this can inadvertently expose allergic consumers to its ingestion. The identification of physical treatments capable of reducing the potential of hazelnut allergens to trigger reactions in sensitized individuals can pave the way for the production of hypoallergenic hazelnut derivatives tolerated by the allergic subjects; this kind of investigation has already been carried out by our team for other nuts, as the case of almonds with an evaluation done in vitro [4]. In the present study we investigated the effects on hazelnut subjected to autoclaving under different schemes, preceded or not by hydration and eventually followed by drying; after obtaining treated hazelnut extracts, we assessed their protein composition, performing gel electrophoresis, western blot and mass spectrometry, and also their skin reactivity, performing a skin prick testing in patients with hazelnut allergy.

\section{Materials and Methods}

\subsection{Patients enrollment for the study}

Twenty-two patients with presumed hazelnut allergy were selected at the University Hospital of Bari (Unit of Allergology). Presumed hazelnut allergy (no oral food challenge was performed to confirm diagnosis) was defined based on allergic symptoms following hazelnut ingestion (such as urticaria, angioedema, allergic oral syndrome, asthmatic symptoms, anaphylaxis) and a positive skin prick test and hazelnut specific serum IgE test [5].

Skin prick tests (SPTs) were carried out on the volar surface of the forearm by puncturing the skin through a droplet of a $100 \mu \mathrm{g} / \mathrm{mL}$ allergen with an $1 \mathrm{~mm}$ point length standardized needle (ALK-Abellò, Milan, Italy). Histamine $10 \mathrm{mg} / \mathrm{mL}$ was used as the positive control. SPTs were performed by placing a drop of extract on the skin of the forearm and subsequently pricking the skin underneath this drop. The test responses were considered positive if the wheal produced had a mean diameter of at least $3 \mathrm{~mm}$. Skin reactivity was also expressed by measuring as the area of the wheals $\left(\mathrm{mm}^{2}\right)$. All the SPT for any allergen were performed in duplicate.

Patients underwent skin testing with 3 different commercial extracts of hazelnut (Lofarma ${ }^{\circledR}, 2 \% \mathrm{w} / \mathrm{v}$, ALK-Abello $\AA, 1: 20 \mathrm{w} / \mathrm{v}$, and Stallergenes ${ }^{\circledR}$ (Alyostal ${ }^{\circledR}$ ), $1 \mathrm{IC} / \mathrm{mL}$ ) and the specifically prepared raw hazelnut seed extracts (Corylus avellana, var. Italiana) at concentration of $2 \mathrm{mg} / \mathrm{mL}$, left untreated (unmodified, denominated A1) or subjected to differential physical treatments (marked as A2, A3 and A4, detailed in the following paragraph).

Saline was used as the negative control of commercial extracts, whereas an urea buffer solution used as diluent for all the hazelnut extracts was used as negative controls for hazelnut extracts.

\subsection{Chemicals}

The study was performed on raw hazelnut seeds (Corylus avellana, var. Italiana) provided by Besana S.p.A. (San Gennaro Vesuviano, Napoli, Italia). Trizma-base, urea, sodium chloride, ammonium bicarbonate (AMBIC), iodoacetamide (IAA), dithiothreitol (DTT), and all chemicals for electrophoresis, namely sodium dodecyl sulfate-SDS, glycine, glycerol, Coomassie brilliant blue-G 250, were provided by Sigma Aldrich (Milan, Italy). Acetonitrile (Gold HPLC ultragradient), Methanol (HPLC grade), trifluoroacetic acid (TFA) and Bromophenol blue were purchased from Carlo Erba Reagents (Cornaredo, Milan, Italia). Ultrapure water was produced by a Millipore Milli-Q system (Millipore, Bedford, MA, USA) while formic acid (MS grade) was purchased from Fluka (Milan, Italy). Polytetrafluoroethylene (PTFE) filters $(0.45 \mu \mathrm{m})$ were obtained from Sartorius (Gottingem, Germania) while syringe filters in cellulose acetate (CA) $1.2 \mu \mathrm{m}$ were purchased from Labochem Science S.r.l. (Catania, Italy). As for in gel protein digestion, trypsin (proteomic grade) was from Promega (Milan, Italy).

\subsection{Hazelnut autoclave treatments}


Autoclaving treatments were accomplished on a total of 3 raw hazelnut kernels (corresponding to approximately $15 \mathrm{~g}$ ) placed into a centrifuge tube. Specifically, three different processing schemes were taken into consideration, namely i) autoclaving, ii) sample pre-hydration followed by autoclaving and iii) sample pre-hydration followed by autoclaving and finally driying in a stove overnight at $60^{\circ} \mathrm{C}$. For the hydration step, $50 \mathrm{~mL}$ of ultrapure water were added to raw hazelnut kernels followed by 2 hours of shaking at room temperature in an orbital shaker (KS 4000 i-control shaker, IKA Works GmbH \& Co. KG, Staufen, Germany). Water was discarded before autoclaving. Autoclaving was accomplished by setting the equipment as following: temperature $134^{\circ} \mathrm{C}$, pressure of $2 \mathrm{~atm}$, cycle time $10 \mathrm{~min}$. The system took about $40 \mathrm{~min}$ to reach the final temperature of $134{ }^{\circ} \mathrm{C}$. The three different schemes investigated are hereafter summarized:

a) Hazelnut autoclaved for $10 \mathrm{~min}(\mathrm{~A} 2)$,

b) Hazelnut prehydrated + autoclaved (A3),

d) Hazelnut prehydrated + autoclaved + driying (A4).

As positive control, raw hazelnut not undergoing any treatment was also included in the study (A1).

The workflow of the proteomic analysis carried out in this work is reported in Figure 1.

\subsection{Protein extraction and quantification}

Before analysis, raw and processed hazelnut seeds were milled by using an electric miller (Mulinex, Milan, Italy) and proteins were extracted by following the protocol described by Bavaro et al., 2018 [6]. Briefly, $10 \mathrm{~mL}$ of TBS buffer (50 mM Tris-HCl, $150 \mathrm{mM}$ $\mathrm{NaCl}, \mathrm{pH} 8+1 \mathrm{M}$ Urea) were added to $0.4 \mathrm{~g}$ of flour and left shaking for $2 \mathrm{~h}$ at room temperature in an orbital shaker (KS $4000 \mathrm{i}$-control shaker, IKA Works GmbH \& Co. KG, Staufen, Germany). The samples were then centrifuged for $15 \mathrm{~min}$ at $1734 \mathrm{x} \mathrm{g}$ at $4^{\circ} \mathrm{C}$ and the supernatant carefully collected while the upper phase discarded. The samples were filtered through $1.2 \mu \mathrm{m}$ CA syringe filters before successive analysis.

The total protein content of raw and autoclaved hazelnut was calculated by Bradford assay (Quick Start ${ }^{\mathrm{TM}}$ Bradford Protein Assay, Bio-Rad Laboratories s.r.l., Segrate MI, Italy) that was carried out according to the manufacturer's instruction. As reference standard the protein bovine serum albumin (BSA, 0.125-1 mg/ml) was used. Samples were stored at $-20^{\circ} \mathrm{C}$ until their use and filtered through $0.45 \mu \mathrm{m}$ PTFE filters just before electrophoretic analysis.

\subsection{Electrophoretic analysis of hazelnut proteins}

Proteins extracted from raw and processed hazelnut samples were then profiled by means of sodium dodecyl sulphate-polyacrylamide gel electrophoresis (SDS-PAGE). Specifically, ten micrograms of proteins were separated under reducing condition on a 8-16\% polyacrylamide pre-cast gel $(13.3 \mathrm{~cm} \times 8.7 \mathrm{~cm} \times 1.0 \mathrm{~mm})$, using a Mini-Protean Tetra Cell equipment (Bio-rad Laboratories, Segrate, MI, Italy). Before electrophoresis analysis, samples were denatured with Laemmli buffer (62.5mM TrisHCl, $\mathrm{pH} 6.8,25 \%$ 


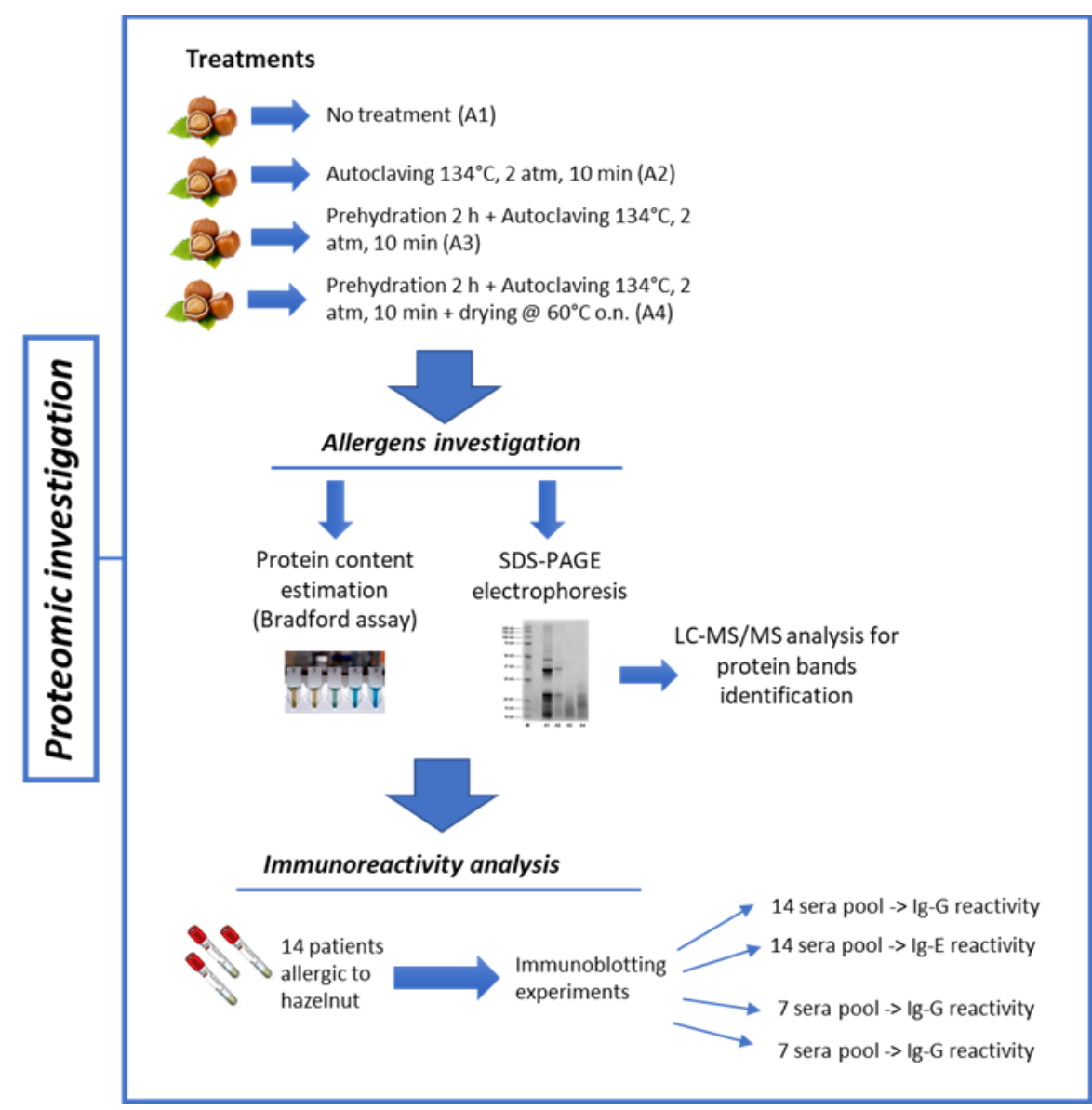

Figure 1. Workflow for proteomic investigation, encompassing extraction step, electrophoretic analysis, protein identification by Mass Spectrometry and immunoblot analysis for immunoreactivity assessment.

glycerol, 2\% SDS, $0.01 \%$ Bromophenol Blue, $100 \mathrm{mM}$ DTT) (1:1 ratio) for $5 \mathrm{~min}$ at $100{ }^{\circ} \mathrm{C}$. Electrophoretic separation was performed at $60 \mathrm{~V}$ for the first $20 \mathrm{~min}$ and then at $100 \mathrm{~V}$ until the end of the run by using a TGS ( $25 \mathrm{mM}$ Tris, $192 \mathrm{mM}$ glycine, $0.1 \%$ SDS) solution as running buffer. Gels were finally stained with Coomassie Brilliant Blue G-250 solution and the protein profiles detected on a ChemiDoc ${ }^{\mathrm{TM}}$ Imaging System (Bio-Rad Laboratories, Segrate, MI, Italy). Precision Plus Protein ${ }^{\mathrm{TM}}$ all blue standards (10-250 kDa, Bio-Rad Laboratories, Segrate, MI, Italy) was used as protein reference for molecular weight.

\subsection{In-gel tryptic digestion and LC-MS analysis}

The most relevant protein bands detected along the electrophoretic gel of raw and treated hazelnut samples were then submitted to in gel digestion for identification purposes. Briefly, the selected bands were excised from the polyacrylamide gels and in-gel trypsin digested according to the protocol described by De Angelis et al., 2017 [7]. After drying, each sample was re-suspended in $100 \mu \mathrm{l}$ of $\mathrm{H}_{2} \mathrm{O} / \mathrm{ACN}$ 95/5+0.1\% formic acid (v/v) and $5 \mu \mathrm{l}$ were injected into LC/MS apparatus.

Peptide mixtures obtained from protein bands in-gel digested referred to samples A1, A2, A3 and A4 were analyzed by untargeted proteomic analysis by HPLC-MS/MS equipment consisting in a Q-Exactive ${ }^{\mathrm{TM}}$ Plus Hybrid Quadrupole-Orbitrap ${ }^{\mathrm{TM}}$ Mass Spectrometer coupled to a UHPLC pump systems (Thermo Fisher Scientific, Bremen, Germany). For the peptide chromatographic separation, a reversed phase Acclaim ${ }^{\mathrm{TM}}$ PepMap100, C18 analytical column $\left(1 \mathrm{~mm} \times 15 \mathrm{~cm} \times 3 \mathrm{~m}, 100^{\circ}\right.$ A porosity, Thermo Fisher Scientific, Bremen, Germany) was used, by setting the flow rate at $60 \mu \mathrm{l} / \mathrm{mL}$. The elution gradient used for peptide separation was the following: 0-60 min solvent B increased from $10 \%$ to $60 \%$, 60$61 \mathrm{~min}$ further increase from $60 \%$ to $80 \%$, then kept constant for $10 \mathrm{~min}, 70-90 \mathrm{~min}$ at a 
constant $10 \%$ for column conditioning before next injection. Solvent $\mathrm{A}=\mathrm{H}_{2} \mathrm{O}+0.1 \%$ FA, solvent $\mathrm{B}=$ Acetonitrile $/ \mathrm{H}_{2} \mathrm{O}(80 / 20 \mathrm{v} / \mathrm{v})+0.1 \%$ FA. Volume injection was set to $5 \mu \mathrm{L}$ and each sample was injected twice in MS. Spectra were acquired in the mass range of 150$2000 \mathrm{~m} / \mathrm{z}$ by applying the data dependent (FullMS-dd2) acquisition mode analysis and only positive ions were considered. Up to 10 most intense ions in MS ${ }^{1}$ were selected for subsequent fragmentation in MS/MS mode. A resolving power of 70,000 full width at half maximum (FWHM), a microscan of 1, an automatic gain control (AGC) target of 1.00 e6 and a maximum injection time (IT) of $40 \mathrm{~ms}$ were set to generate precursor spectra (full MS analysis). The settings for $\mathrm{MS}^{2}$ fragmentation experiments were the following: resolving power 17,500 FWHM, microscan of 1, AGC target $5.00 \mathrm{e}^{5}$, maximum IT $50 \mathrm{~ms}$, loop count 10, MSX count 1, isolation window of $2.0 \mathrm{~m} / \mathrm{z}$, isolation offset $0.4 \mathrm{~m} / \mathrm{z}$ and normalized collision energy (NCE) at 27 and $30 \mathrm{eV}$ by activating the stepped option; as for $\mathrm{dd}$ settings maximum AGC target was set at $5.00 \mathrm{e}^{1}$ with intensity threshold of $1.0 \mathrm{e}^{3}$, dynamic exclusion at $20 \mathrm{~s}$, peptide match set to preferred and exclude isotopes enabled. All ions with charge equal to 1 and higher than 4 were excluded. As for HESI source, the following conditions were set: spray voltage 3400 , capillary $\mathrm{T}^{\circ} \mathrm{C} 320$, sheat and auxiliary gas 25 and 15 respectively, probe heater $\mathrm{T}^{\circ} \mathrm{C} 290$ and S-Lens 55.

Raw MS data were then processed via the commercial software Proteome Discoverer ${ }^{\mathrm{TM}}$ version 2.1.1.21 (Thermo-Fisher-Scientific, Bremen, Germany) and protein identification was obtained by SequestHT search against the hazelnut proteins database extracted by Swiss Prot DB on the base of the taxonomy code of Corylus avellana (ID: 13451, containing about 501 sequences). Trypsin was selected as cleavage enzyme and in all cases mass tolerance on the precursor and fragment ions was set to $5 \mathrm{ppm}$ and $0.05 \mathrm{Da}$, respectively. Finally, only trustful peptide-spectrum matches were taken into account and a minimum of three peptides was set as threshold for protein identification, after filtering the peptide list to the sequences assigned with at least medium confidence (FDR $<5 \%)$.

\subsection{Immunoblot for IgE-Binding Assay}

Proteins of raw and differently treated hazelnuts separated by SDS-PAGE (corresponding to $10 \mu \mathrm{g}$ of proteins loaded for A1, A2, A3 and A4) were electroblotted onto a $0.2 \mu \mathrm{m}$ nitrocellulose membrane (Bio-Rad Laboratories, Segrate, MI, Italy) using a TransBlot Cell (Bio-Rad Laboratories, Segrate, MI, Italy) for 7 min (1.3 A, 25 V). Immunoblotting tests were accomplished according to the protocol reported by Bavaro et al., 2019 [8]. As primary antibody, the pooled sera of a total of 14 allergic individuals showing different clinical symptoms (urticaria, itching, hands/throat angioedema) previously diluted in TBS-T (pH 7.4, $10 \mathrm{mM}$ Tris, $50 \mathrm{mM} \mathrm{NaCl}, 0.1 \%$ Tween 20) at a 1/50 ratio was used and kept shaking overnight at $4{ }^{\circ} \mathrm{C}$. As a secondary antibody goat anti-human $\operatorname{IgG}(\mathrm{H}+\mathrm{L})$ horseradish peroxidase (HRP) conjugated (Bio-Rad Laboratories) and anti-human IgE $(\varepsilon-$ chain specific)-horseradish peroxidase conjugated (Sigma Aldrich, Milan Italy) each diluted $1 / 5000(\mathrm{v} / \mathrm{v})$ in TBS-T were added. Specifically, four different immunoblotting experiments were accomplished: in the first two experiments two different sera pools, each obtained by combining the sera of 7 patients per group, were used and the reactive bands detected by using the goat anti-human $\operatorname{IgG}(\mathrm{H}+\mathrm{L})$ horseradish peroxidase (HRP) conjugate (vide infra) as secondary antibody. In the third and fourth experiments, the sera of all 14 patients were pooled together and the reactive proteins were identified after incubation with anti-human IgE ( $\varepsilon$-chain specific)-peroxidase secondary antibody binding and goat anti-human IgG $(\mathrm{H}+\mathrm{L})$ horseradish peroxidase $(\mathrm{HRP})$ (vide infra). Final images were obtained on a ChemiDoc ${ }^{\mathrm{TM}} \mathrm{MP}$ Imaging System.

\subsection{Statistical analysis}

T-test was used to compare the average areas of the wheals.

\section{Results and Discussion}

\subsection{Effects of hazelnut thermal/pressure treatment on patient response}


Firstly, we sought to assess whether the modified hazelnut extracts were associated with a reduced patients' response in vivo. To this aim, 22 patients with diagnosed hazelnut allergy (16 females [72.7\%]; mean age, $28.1 \pm 9.4$ years; range: 17-48 years) underwent skin prick testing [9]. Specifically, 19 (86.4\%) out of 22 patients reported urticaria/angioedema, 3 patients reported oral allergy syndrome (13.6) and 1 patients reported both manifestations $(4.5 \%)$, suggesting that most patients were likely sensitized to hazelnut proteins, like Cor a 8 , Cor a 9, Cor a 11, that are known to be responsible for systemic symptoms (Table $1)$.

Table 1. Hazelnut allergens. Overview of different protein families, MWs and specific related clinical pictures.

\begin{tabular}{llll}
\hline Protein name & Protein family & MW (kDa) & Clinical picture \\
\hline Cor a 1.04 & PR-10 (Bet v 1 like) & 17 & OAS \\
Cor a 2 & profillin (Bet v 2 like) & 14 & OAS \\
Cor a 8 & LTP & 9 & Systemic reactions \\
Cor a 9 & SSP (11S globulin - legumin like) & 40 & Systemic reactions \\
Cor a 11 & SSP (7S globulin - vicilin like) & 48 & Systemic reactions \\
Cor a 12 & Oleosin & 17 & \\
Cor a 13 & Oleosin & $14-16$ & \\
Cor a 14 & SSP (2S albumin) & 10 & \\
\hline
\end{tabular}

The SPT reactivity (defined as the mean wheal area of 2 independent measurements) reported with the hazelnut extract A1 (unmodified hazelnut seed extracts - Corylus avellana, var. Italiana) was $24.6 \pm 12.3 \mathrm{~mm}^{2}$, comparable to that produced by the 3 commercial extracts (ALK, $26.5 \pm 13.2 \mathrm{~mm}^{2}$; Stallergenes, $22.8 \pm 12.3 \mathrm{~mm} 2$; Lofarma, $18.7 \pm 9.0 \mathrm{~mm}^{2}$. This suggested that the native extract A1 was effective and suitable to be used for comparisons with modified extracts. Negative controls (either saline or urea solutions) did not elicit any skin reaction (no wheal, no erythema, no pruritus).

Only 6 patients (27.3\%) showed reactivity with the A2 extract. This reactivity appeared lower than that of the native extract (mean wheal area of the 6 reactive patients, $15.2 \pm 4.1$ $\mathrm{mm}^{2}$; P [A1 vs. A2] = 0.005) (Figure 2; Table 2).

Only 2 out of the 6 patients reactive to A2 showed reactivity with the A3 extract. The mean wheal area of the 2 reactive patients was $13.5 \mathrm{~mm}^{2}$ (patient \#15) e $4.5 \mathrm{~mm}^{2}$ (patient \#20), respectively (Figure 2; Table 2).

Finally, only 1 patient (patient \#15) showed SPT reactivity (wheal area, $13.5 \mathrm{~mm}^{2}$ ) with the A4 extract.

Collectively, these results suggest that all the 3 different treatments are effective in reducing hazelnut allergenicity in a meaningful proportion of patients. Treatments 3 and 4 appeared to be the most effective ones. Notably, one patient out of 22 did not show a significant reduction of SPT reactivity even with the A4 extract. Further analyses will be necessary to define the specific epitopes this patient is reactive to. 


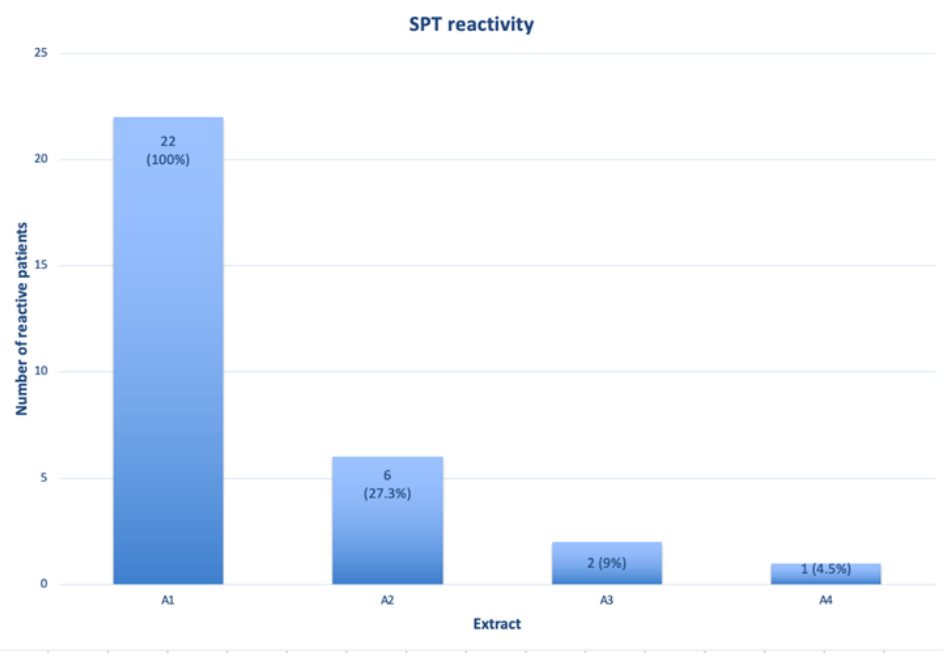

Figure 2. Number and percentage of patients with SPT positive to differentially treated hazelnut extracts. Number and percentage (in brackets) of reactive patients are reported in each columns.

Table 2. SPT reactivity of patients with differentially treated hazelnut extracts.

\begin{tabular}{llll}
\hline Extract & $\begin{array}{l}\text { \# reactive pa- } \\
\text { tients } \\
\text { N (\%) }\end{array}$ & $\begin{array}{l}\text { Wheal area, } \mathbf{m m}^{2} \\
\text { (mean } \pm \text { SD) }\end{array}$ & $P$ \\
\hline A1 & $22(100 \%)$ & $24.6 \pm 13,1$ & Reference \\
A2 & $6(27.3)$ & $15.2 \pm 4.1^{2}$ & p $<0,01$ \\
A3 & $2(9.1)$ & 13.5 (patient \#15) & n.e. ${ }^{1}$ \\
A4 & $1(4.5)$ & 4.5 (patient \#20) & \\
\hline
\end{tabular}

${ }^{1}$ n.e $=$ not estimable

${ }^{2}$ mean \pm SD calculated with data from only the 6 reactive patients; reference means that this group (A1) is used for comparisons with the other groups (A2, A3, A4) for statistical analyses.

\subsection{Effect of thermal/pressure treatment on hazelnut protein solubility}

Food processing is known to alter the final structure and function of proteins, modifying some crucial properties such as the final solubility. Denaturation, hydrolysis of peptide bonds, restructuring of disulphide bonds and interaction with other components (carbohydrates and lipids) that frequently could occur during treatments are among the causes leading to reduced protein solubility [10]. In the light of this, we first sought to evaluate how autoclaving/pressure processing could alter the final solubility of hazelnut proteins by estimating the total protein content of samples undergoing the different treatments. The Bradford assay was used for this purpose (Figure 3). A progressive reduction in protein recovery was shown in the treated hazelnut samples compared to the untreated counterpart. The total protein content of the autoclaved samples (A2) appeared to be $40 \%$ lower than control sample (A1), with a more dramatic reduction observed for pre-hydrated/autoclaved sample (A3) and pre-hydrated/autoclaved/dried samples (A4), which showed a $70 \%$ decrease in protein recovery compared to the untreated hazelnut sample (Figure 3). 


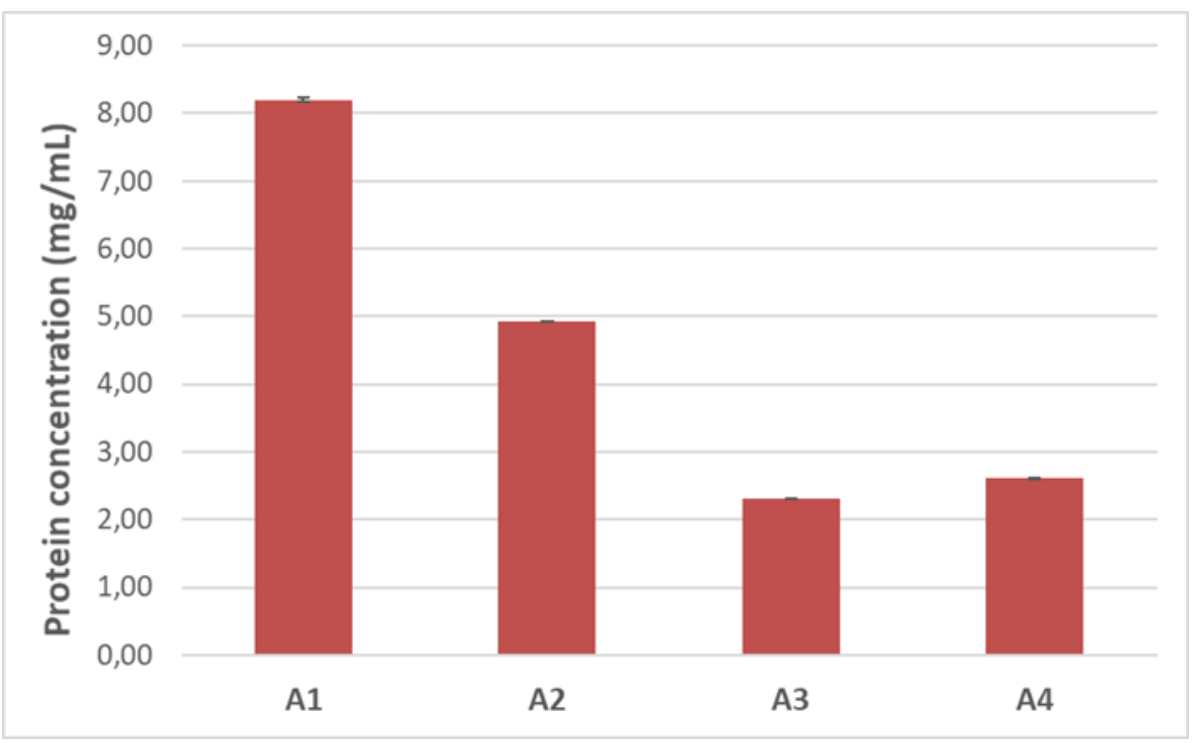

Figure 3. Protein content, estimated by Bradford protein assay, corresponding to untreated hazelnut (A1) and hazelnut submitted to autoclaving (A2), prehydration/autoclaving (A3) and prehydration/autoclaving followed by drying (A4).

These results are in accordance with previous studies, reporting the effects of autoclaving on other nuts species, such as almonds or peanuts $[4,6,11,12]$. In general, it was demonstrated that autoclave based treatments produce a decrease in protein recovery and this trend is more marked when treatment is accomplished at harsher conditions or when it is preceded by a hydration step. This phenomenon could be attributed to the numerous biochemical and structural modifications occurring on protein moiety taking place during the combination of heat and pressure treatments. The latters, in combination with aggregation phenomena due to the formation of intra- or inter-molecular covalent and noncovalent interactions between proteins or protein-food matrix, could promote protein precipitation with a consequent decrease in protein solubility and protein content in the final extract [13]. As for hazelnut, Lopez et al. in 2012 demonstrated that autoclaving could alter the secondary and tertiary structure of this tree nut proteins also inducing a glycosylation reaction [14]. In the current study, the reduced protein content observed in treated hazelnuts confirmed that autoclave-based treatments could modify the protein structure of hazelnut proteins, leading to a reduction of their solubility. This effect appears to be enhanced by preceding autoclaving with water incubation. In addition to a reduced solubility, a fragmentation of the allergen protein is also likely to occur during autoclaving due to the pressure and temperature applied. It is already known that according to some OFC studies $[15,16]$ new thresholds might be proposed for allergen undergoing heating / baking treatments in food matrices as they can induce modification of protein structure and decrease in its allergenicity. In line with this, it is reasonable that the reduced protein extraction or protein degradation after physical treatments might have influenced the overall SPT reactivity.

\subsection{SDS-PAGE profiling of processed hazelnuts and proteins identifications}

Raw (A1) and differently processed hazelnuts (A2, A3 and A4) were electrophoretically profiled in order to evaluate possible changes in hazelnut protein content and structure induced by the different treatments. Specifically, $10 \mu \mathrm{g}$ of untreated (A1) and treated hazelnut proteins (A2, A3 and A4, respectively) were analyzed by SDS-PAGE and peculiar protein profiles were observed for each sample analyzed (Figure 4). In the untreated 
sample (Figure 4A, lane A1), several bands in the region of 30 - $50 \mathrm{kDa}$ and 10 - $22 \mathrm{kDa}$ were shown. As known from the literature, in the absence of reducing agents, Cor a 9 (11S legumins) is organized in hexameric structures made up of six subunits interacting noncovalently and arranged in an open ring conformation with $360 \mathrm{kDa}$ [17].
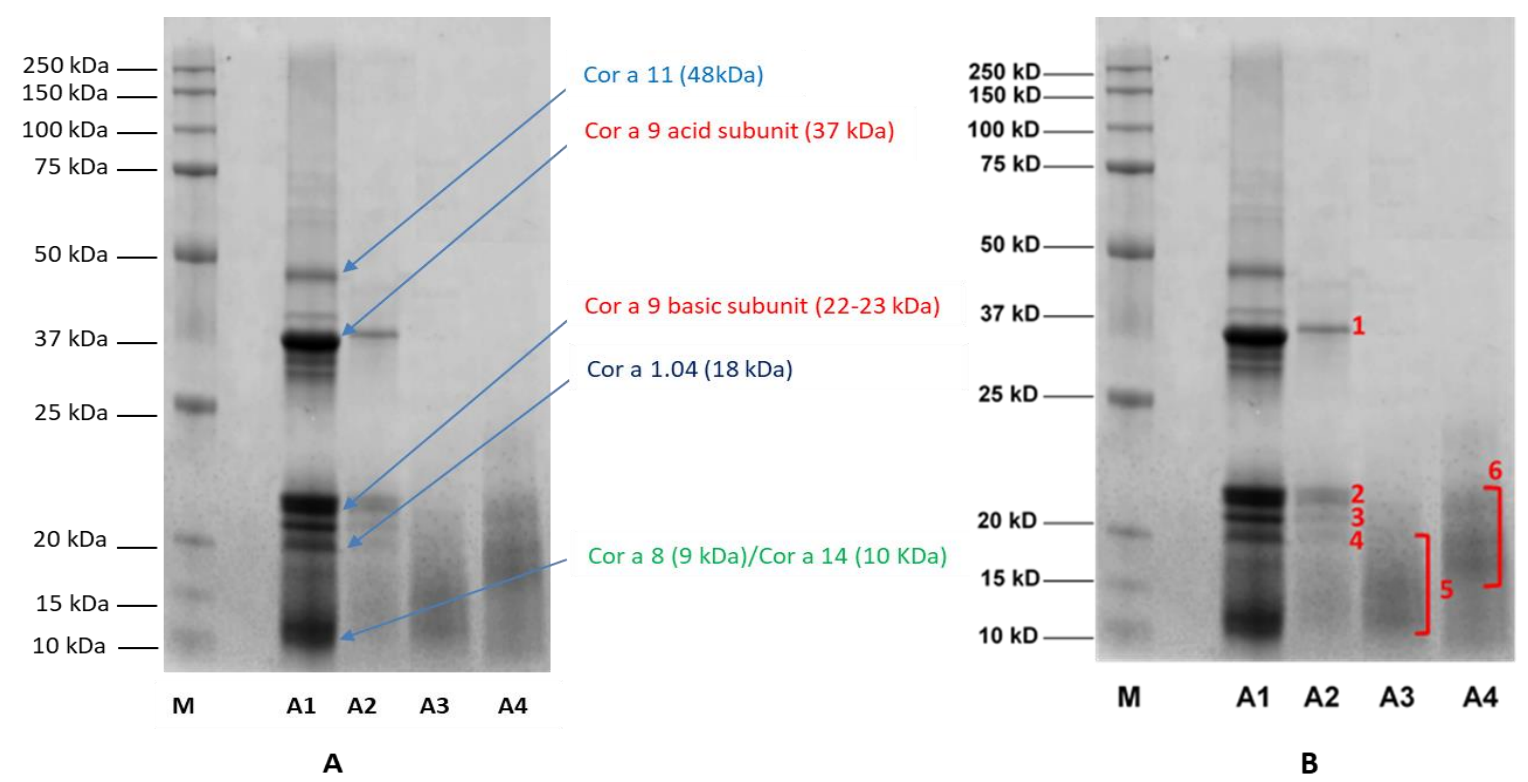

Figure 4. A) Comparison between SDS-PAGE protein profiles of hazelnut untreated (A1), autoclaved (A2), prehydrated and autoclaved (A3), prehydrated/autoclaved and subsequently dried (A4). M, MW reference standards. B) Protein bands excised from the gel (numbered from 1 to 6) to be submitted to tryptic digestion and analysis by untargeted High Resolution Mass Spectrometry.

Each subunit is composed by an acidic polypeptide (30-40 kDa) linked to a basic polypeptide (around $20 \mathrm{kDa}$ ) by a disulphide bond [18]. Under reducing conditions, acid and basic subunits are released and they are clearly visible in the lane of the untreated sample (Figure $4 \mathrm{~A}$, lane A1). Other bands were visible over the $37 \mathrm{kDa}$ region and below $20 \mathrm{kDa}$. They are likely to be attributed to Cor a 11, Cor a 8 and Cor a 14, whose MWs were reported to be approximately $48 \mathrm{Da}, 9 \mathrm{kDa}$ and 15-16 kDa, respectively [18]. After autoclaving (Figure 4 A, lane A2), a general decrease in bands intensity was recorded, with a concomitant disappearance of the proteins banding at $50 \mathrm{kDa}$ and below $20 \mathrm{kDa}$ that were putatively attributed to Cor a 11, Cor a 8 and Cor a 14 allergens. As for Cor a 9, a marked reduction of signals corresponding to acid and basic subunits was observed, likely attributable to a reduced content of the allergen following the thermal/pressure treatment applied. On the contrary, protein profiles of hazelnut samples incubated with water before autoclaving (Figure 4 A, lanes A3 and A4) appeared as a smear of peptides, with low MW (10-20 kDa), probably produced by fragmentation occurring during the treatments. It is worthy to be noted that drying process after autoclaving (A4 treatment) did not produce any significant difference in protein profile with respect to the A3 sample.

SDS-PAGE profiles are in accordance with protein assay results (Figure 3), where a progressive reduction in protein content was displayed among the hazelnut samples differently treated, with a more marked decrease observed for pre-hydrated/autoclaved and pre-hydrated/autoclaved /dried samples. 
To gain insights on the protein content of the specific bands identified by SDS-PAGE, the most relevant bands, which resulted differently expressed after different treatments (Figure $4 \mathrm{~B}$, lanes $\mathrm{A} 2, \mathrm{~A} 3$ and $\mathrm{A} 4$ ) were excised from the gel (the excised bands are numbered from 1 to 6 ), submitted to tryptic digestion and analyzed by untargeted High Resolution Mass Spectrometry. MS spectra were then processed by Proteome Discoverer software for protein identification. Specifically, the Uniprot database referred to Corylus avellana (last accession on $26^{\text {th }}$ of November 2021) was interrogated and results are resumed in Table 3.

Table 3. Summary of proteins identified by Proteome Discoverer software referred to SDS-PAGE protein bands excissed and in gel digested from protein pattern of autoclaved (A2), prehydrated/autoclaved (A3), prehydrated/autoclaved/dried (A4) hazelnut samples.

\begin{tabular}{|c|c|c|c|c|c|c|c|}
\hline Sample & Band & Accession & Description & Allergen & $\begin{array}{c}\text { Coverage } \\
(\%)\end{array}$ & $\begin{array}{l}\text { \#Peptides } \\
\text { (unique) }\end{array}$ & Score \\
\hline \multirow{8}{*}{ A2 } & \multirow{2}{*}{1} & A0A0A0P7E3 & Cor a 9 allergen (Corylus avellana) & Cor a 9 & 52 & $21(2)$ & 124 \\
\hline & & Q8W1C2 & 11S globulin-like protein (Corylus avellana) & Cor a 9.0101 & 45 & $23(2)$ & 112 \\
\hline & \multirow{2}{*}{2} & A0A0A0P7E3 & Cor a 9 allergen (Corylus avellana) & Cor a 9 & 44 & $17(4)$ & 207 \\
\hline & & Q8W1C2 & 11S globulin-like protein (Corylus avellana) & Cor a 9.0101 & 37 & $14(1)$ & 202 \\
\hline & \multirow{3}{*}{3} & A0A0A0P7E3 & Cor a 9 allergen (Corylus avellana) & Cor a 9 & 40 & $16(3)$ & 118 \\
\hline & & Q8W1C2 & 11S globulin-like protein (Corylus avellana) & Cor a 9.0101 & 33 & $14(1)$ & 116 \\
\hline & & A0A1I9RG40 & $\begin{array}{l}\text { Ribulose bisphosphate carboxylase large } \\
\text { chain (Corylus avellana) }\end{array}$ & & 19 & $8(8)$ & 16 \\
\hline & 4 & A0A0A0P7E3 & Cor a 9 allergen (Corylus avellana) & Cor a 9 & 28 & $11(11)$ & 38 \\
\hline \multirow{5}{*}{ A3 } & \multirow{5}{*}{5} & Q8W1C2 & 11S globulin-like protein (Corylus avellana) & Cor a 9.0101 & 32 & $18(2)$ & 111 \\
\hline & & A0A0A0P7E3 & Cor a 9 allergen (Corylus avellana) & Cor a 9 & 34 & $19(2)$ & 109 \\
\hline & & & & Cor a & & & \\
\hline & & D0PWG2 & 2S albumin (Corylus avellana) & $14 /$ Cor a & 44 & $8(8)$ & 15 \\
\hline & & & & 14.0101 & & & \\
\hline \multirow{6}{*}{$\mathrm{A} 4$} & \multirow{6}{*}{6} & A0A0A0P7E3 & Cor a 9 allergen (Corylus avellana) & Cor a 9 & 30 & $14(2)$ & 98 \\
\hline & & Q8W1C2 & 11S globulin-like protein (Corylus avellana) & Cor a 9.0101 & 28 & $14(2)$ & 98 \\
\hline & & D0PWG2 & $2 \mathrm{~S}$ albumin (Corylus avellana) & $\begin{array}{c}\text { Cor a } \\
14 / \text { Cor a } \\
14.0101\end{array}$ & 31 & $8(8)$ & 4 \\
\hline & & & & Cor a & & & \\
\hline & & Q8S4P9 & Vicilin Cor a 11.0101 (Corylus avellana) & $11 /$ Cor a & 8 & $2(2)$ & 2 \\
\hline & & & & 11.0101 & & & \\
\hline
\end{tabular}

As expected, bands 1 and 2 of the autoclaved sample (Figure 4 B, lane A2) were attributed to Cor a 9 allergen, suggesting the resistance of this allergen to autoclave processing although the reduced intensity of the relative bands leads to supposing that some partial degradation/structural alteration of the proteins likely occurred during the treatment. Cor a 9 allergen was found also in bands 3 and 4 (Figure 4 B, lane A2). The smeared bands visible in protein profiles of pre-hydrated/autoclaved samples followed or not by drying (approximately 10-20 kDa, bands 5 and 6, respectively) (Figure 4 B, lanes A3 and A4) were instead produced by a mix of peptides belonging to Cor a 9, Cor a 11 and Cor a 14 allergens (Table 3). 
The impact of autoclaving on protein stability of tree nuts and peanuts was already reported in the literature $[4,6,14,19,20]$, with some papers exploring the effect of water incubation before thermal/pressure treatment $[4,6]$. Similar to what described in the present work, these authors observed that samples pretreated with water before autoclaving showed a SDS-PAGE protein profile more fragmented and degraded with respect the autoclaved counterparts. To explain these phenomena, they suggested that probably water absorbed by seeds during incubation facilitates the propagation of heat in the inner part of the seed promoting protein disaggregation with consequent decrease in band intensity. In addition, our results are in line with what was described by Lopez et al. in 2012, who studied the effect of different conditions of autoclaving and high-pressure treatments on the final allergenicity of hazelnut flour. The authors highlighted the disappearance of the main hazelnut allergens protein bands in SDS-PAGE profile of hazelnuts autoclaved at $131^{\circ} \mathrm{C}$ for 15 or $20 \mathrm{~min}$, ascribing these results to molecular alterations or post- translational modifications (PTMs, e.g. glycosylation) that likely occur during autoclaving. Indeed, by generating a series of bioinformatics homology-based 3D models, they found that the structure of the main hazelnut allergens (Cor a 8, Cor a 9) resulted altered after autoclaving with a new site of glycosylation found in Cor a 11 allergen [14].

\subsection{Immunoblotting experiments}

To assess the immunogenicity and the allergenicity of hazelnut seeds subjected to the different autoclaving treatments (A2, A3, A4), immunoblotting experiments were performed. Sera from 14 patients showing different clinical symptoms, both systemic and OAS, were used for immunoblotting experiments (Figure 5). 

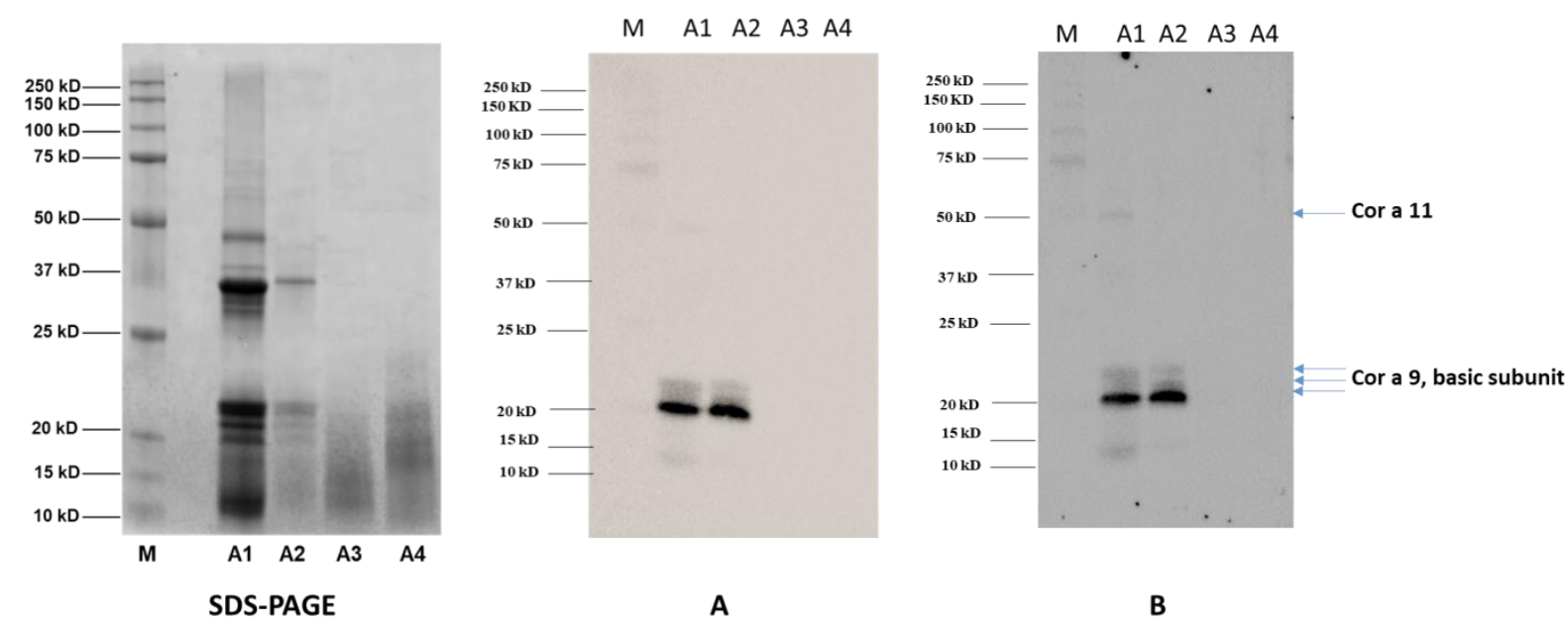

Figure 5. SDS-PAGE along with Immunoblots of hazelnut untreated (A1) or submitted to autoclave (A2), pre-hydration/autoclave (A3), prehydration/autoclave/drying (A4). M, Precision Plus Protein $^{\mathrm{TM}}$ All Blue Standard (10-250 kDa, Bio-Rad Laboratories, Segrate, Milan, Italy). Panel A and B: immunoblotting profiles of 14 patients allergic to hazelnut pooled together and incubated with goat anti-rabbit IgG Ab (Panel A) and anti-human IgE Ab ( $\varepsilon$-chain specific) secondary antibody (Panel B). Panel C and D: immunoblotting profiles of two subgroups of 14 allergic patients ( 7 allergic sera pooled together for each group) both incubated with goat anti-rabbit IgG Ab.

The pooled sera of a total of 14 allergic patients were used as the primary antibody. Then, the membranes were incubated with a goat anti-rabbit IgG Ab (Figure $5 \mathrm{~A}$ ) or an antihuman $\operatorname{IgE} \mathrm{Ab}$ ( $\varepsilon$-chain specific) (Figure $5 \mathrm{~B}$ ), as the secondary antibodies. Finally, to increase the assay sensitivity, 2 additional subgroups with 7 pooled sera per group (Figure $5 \mathrm{C}-\mathrm{D}$ ) were incubated with a goat anti-rabbit IgG Ab (vide infra). Figure $5 \mathrm{~A}$ shows in untreated hazelnut (lane A1) one main reactive band with MW of approximately $20 \mathrm{kDa}$ visible, along with weak intensity bands at $50 \mathrm{kDa}$ and in the range of 10 and $15 \mathrm{kDa}$. Two additional blurred signals between 21-22 $\mathrm{kDa}$ were displayed in the A1 sample as well. According to the literature, protein banding at $20 \mathrm{kDa}$ could be putatively attributed to the basic subunit Cor a 9 hazelnut allergens as well as bands in the range of 21-22 kDa. On the contrary, the reactivity displayed at $50 \mathrm{kDa}$ could be ascribable to Cor a 11 allergen, which original MW is $48-50 \mathrm{kDa}$, while the weak signal in the range of 10-15 $\mathrm{kDa}$ could be attributed to Cor a 8 or Cor a 14 allergens with MWs falling in this range. After autoclaving treatment (Figure $5 \mathrm{~A}$, lane A2), the weak reactivity of signals at $50 \mathrm{kDa}$ and below $15 \mathrm{kDa}$ became negligible, while the intense spot at $20 \mathrm{kDa}$ and bands in the range of $21-22 \mathrm{kDa}$ still persisted. Grounding on proteomic investigation accomplished on SDS-PAGE protein profile, these bands were attributed to Cor a 9 and its isoform allergen Cor a 9.0101 (Table 3 ), confirming that these allergens survived to autoclave processing preserving its allergic 
potential. On the contrary, no reactive signal was displayed for the band at $50 \mathrm{kDa}$, putatively ascribed to Cor a 11, along with the 10-15 kDa hazelnut allergens (likely Cor a 8 and Cor a 14), confirming the susceptibility of these proteins to autoclave processing (Figure $5 \mathrm{~A})$. As for the prehydrated/autoclaved (Figure 5 A, lane A3) and prehydrated/autoclaved/dried (Figure $5 \mathrm{~A}$, lane A4) samples, no clearly identifiable reactive bands were shown, thus confirming the key role of the water imbibition step in the structural and conformational alteration/degradation phenomena induced and enhanced by the ther$\mathrm{mal} /$ pressure treatment applied on proteins. Cor a 9 was demonstrated to be a highly wellstructured protein, enriched with a beta-sheet core and with long unstructured loops. These loop regions were found structurally unstable and were predicted to retain linear epitopes located at the external faces of protein, thus being exposed to solvent [14]. After submitting hazelnut samples to autoclaving $\left(121^{\circ} \mathrm{C}\right.$ or $138^{\circ} \mathrm{C}$ for 15 or $\left.30 \mathrm{~min}\right)$, Lopez and co-workers obtained a reduction of the Cor a 9 allergenicity, since no bands corresponding to this protein was visible by SDS-PAGE analysis. In the light of this, the authors hypothesized that the allergenicity of Cor a 9 could be predominantly ascribable to structural conformation and not to linear epitopes [14]. Our results seem to support this hypothesis. Indeed, the enhanced action of previous water incubation on autoclaving (A3, A4) seems effective in impairing the Cor a 9 structure with no reactive epitopes surviving after processing, differently from with what was observed for the solely autoclaved sample (Figure 5 A, lane A2). By visual inspection of the SDS-PAGE pictured in figure 4B, despite the visible smear bands below $20 \mathrm{kDa}$, (produced by a mixture of Cor a 9 and Cor a 14 allergens peptides in A3 sample and Cor a 9, Cor a 14 and Cor a 11 allergens peptides in A4 sample as highlighted by proteomic investigation in Table 3), no reactive signals were displayed in the putatively corresponding lanes of immunoblotting profiles. As already emerging from the electrophoretic analysis, no significant changes in immunoblotting profile and consequently in the final allergenicity of prehydrated/autoclaved hazelnut were found by drying the sample (Figure 5, lane A3).

Similar results were obtained by incubating the A1-A4 samples with anti-human IgE Ab (vide infra) ( $\varepsilon$-chain specific) secondary antibody, confirming that specific IgE can bind protein bands detected in untreated and autoclaved samples and, thus, likely trigger an allergic reaction in vivo. On the contrary, no IgE reactivity was displayed for A3-A4 samples pointing out their likely lack of allergic potential.

Considering that sensitization to specific hazelnut proteins could vary among allergic individuals, additional immunoblotting experiments were performed dividing the 14 patient sera in 2 groups of 7 patient sera. Our aim was to unveil possible different pattern of sensitization of different patient subgroups. In particular, Figure $5 \mathrm{C}$ and D shows immunoblotting experiments conducted using 2 different sera pools each of which made of 7 patients' sera. These immunoblots showed different sensitivity compared to those showed in Figure $5 \mathrm{~A}$ and B (using the pool made by all the 14 patients), depending on the differential antibody concentration of each patient's serum in the subgroups.

As a matter of the fact, the reactivity profile varied among the different pools. Figure $5 \mathrm{C}$ showed more intense bands at approximately $50 \mathrm{kD}$ (Cor a 11), $37 \mathrm{kD}$ (Cor a 9 acid subunit), 20-22 kD (Cor a 9 basic subunit/Cor a 1.04) and $10 \mathrm{kDa}$ (Cor a 8/Cor a 14), compared to Figure 5 A, B and D. This was expected, considering that pools represent patients' average response, which can vary along with the differential pool array. Further analyses on individual patient sera will be necessary to accurately identify the individual reactivity profile, and how this could be modified by specific treatments.

The effect of the autoclaving on the final allergenicity of hazelnuts was already investigated in 2012 by Lopez et al. The authors submitted hazelnut defatted flour to autoclave processing at different conditions $\left(121^{\circ} \mathrm{C} 15 \mathrm{~min}, 121^{\circ} \mathrm{C} 30 \mathrm{~min}, 138^{\circ} \mathrm{C} 15 \mathrm{~min}, 138^{\circ} \mathrm{C} 30\right.$ min) and investigated the IgE-reactivity of 15 allergic patients via Western blot experiments. In addition, they studied the changes induced by autoclaving to the conformational structure of hazelnut allergens by generating a series of homology-based-bioinformatics 3D-models for allergens Cor a 1, Cor a 8, Cor a 9 and Cora 11. As result, the authors observed that at harsher conditions $\left(138^{\circ} \mathrm{C} 15 \mathrm{~min}, 138^{\circ} \mathrm{C} 30 \mathrm{~min}\right)$ autoclaving induced a 
severe reduction of hazelnut allergenicity in the patients studied. Indeed, the specific-IgE binding of some immunoreactive hazelnut protein-bands such as Cora 1, Cora 8, Cora 9 and Cora 11 (vide infra) decreases. Moreover, the structural analysis (3D-modelling) of these allergens highlighted that a relevant glycosylation occurred in the protein-allergen Cora 11 (vide infra) after autoclaving, pointing out that the combination of temperature/pressure could promote the interaction of protein-matrix, likely altering the final allergenicity of the protein [14].

Very recently Cuadraro et al. investigated the effect of autoclaving on the final allergenicity of Cor a 9, Cor a 14 and Cor a 8 hazelnut allergens by testing the two different autoclaving temperatures, $121^{\circ} \mathrm{C}$ and $138^{\circ} \mathrm{C}$, for $30 \mathrm{~min}$. Whole hazelnut seeds were processed and, similarly to what described by Lopez et al., they observed in the different immunoblot profiles a marked reduction of Cor a 9, Cor a 14 and Cor a 8 reactivity after autoclaving hazelnut material at $138^{\circ} \mathrm{C}$ for $30 \mathrm{~min}$ [21].

In the present investigation we observed that ,by autoclaving hazelnut seeds at $134^{\circ} \mathrm{C}$ for $10 \mathrm{~min}$, the intensity of Cor a 9 band appeared to be reduced, with the disappearance of the $50 \mathrm{kDa}$ and $10 \mathrm{kDa}$ bands, putatively ascribed to Cor a 11 and Cor a 8 allergens, as displayed in the SDS-PAGE picture (Figure 3, lane A2). These results are comparable to what obtained by the mentioned authors by applying similar autoclaving conditions (temperature of $121^{\circ} \mathrm{C}$ for $30 \mathrm{~min}$ ) [14, 21].

Furthermore, the immunoblot profiles presented here confirmed the IgG and the IgE reactivity of Cor a 9 basic subunits after the autoclaving of hazelnut material only (Figure 5, panel A-D). On the contrary, by incubating hazelnut seeds with water before autoclaving a full degradation/fragmentation of the proteins was observed, with the disappearance of the main allergenic bands in the corresponding SDS-PAGE (Figure 3, lane A3 and A4) and immunoblotting profile (Figure 5, panel A-D). These results are comparable with what observed by Lopez et al. and Cuadraro et al. by analyzing hazelnut autoclaved at $138^{\circ} \mathrm{C}$ for $30 \mathrm{~min}$ via SDS-PAGE and Western blot experiments [14,21]. In the light of this, it is reasonable to suppose that water incubation strengthens the alterative phenomena induced by autoclaving on proteins allowing to produce the same effect obtained by autoclaving hazelnut at very harsh conditions, such as $138^{\circ}-30 \mathrm{~min}$.

\section{Conclusions}

This preliminary study shows that autoclave treatments, also preceded by hydration and/or followed by drying, appears to be effective in reducing the allergenicity of hazelnut proteins in most of the patients herein screened by skin prick test, probably due to denaturation/fragmentation of most major and minor allergenic proteins. The reduction of hazelnut proteins solubility due to the specific treatment applied must be also considered in the interpretation of the reduced allergenicity proved by immunoblot tests. Anyway, taking into account that immunoblotting experiments were accomplished by loading the same amount of proteins for all treated samples, the reduction of reactivity in the observed bands could be reasonably attributed to a reduced allergenicity of those proteins. The appearance of a smear corresponding to low MW proteins (lower than $20 \mathrm{kDa}$ ) along with the absence of higher MW bands also suggests a possible protein fragmentation caused by physical treatments, and this could be responsible for both reduced IgG and IgE reactivity. Furthermore, unique peptides corresponding to Cor a 9 and Cor a 11 were detected in the low MW degraded proteins (protein bands 5 and 6 in Figure 4 B and Table 3) confirming the hypothesis of protein degradation. Notably, Cor a 9 and Cor a 11 are two of the three most important and harmful hazelnut allergens causing systemic reactions. The third allergen causing systemic reaction, Cor a 8, was likely present in the band approximately at $10 \mathrm{kDa}$. However, a possible fragmentation of this protein can only be hypothesized, but not demonstrated, because the fragments resulting from the effects of each treatment would be too small to be identified with our immunoblots as they could have escaped the gel meshes during gel running. Further specific experiments will be performed to confirm this hypothesis. Nonetheless, according to studies based on oral food 
challenges, threshold doses below which reactions do not occur exist for allergens. Consequently, it is possible that the reduced protein extraction after physical treatments might have influenced SPT reactivity. Whether the reduced SPT reactivity that we observed in our patients could be correlated to a decreased response to Oral food challenge (OFC) needs to be elucidated and will be investigated in further studies.

In conclusion this study, based on patients' reactivity in vivo (SPT) and their immunoblotting profiles, showed that specific physical treatments can reduce the allergenicity of hazelnut. These results need to be confirmed by double blind placebo-controlled food challenge with the treated extracts. Finally, in the perspective of using these physical treatments in food production, the effects of them on the organoleptic properties of the preparation (smell, taste) is also worthy to be further investigated and optimized for commercialization purposes.

Aknowledgements:

The authors are thankful to Simona Bavaro for helping in running immunoblotting analysis, Roberto Schena for the technical aid and Mariella Quarto for the administrative help (ISPA-CNR).

Supplementary Materials: The following supporting information can be downloaded at: www.mdpi.com/xxx/s1, Figure S1: title; Table S1: title; Video S1: title.

Author Contributions: Conceptualization, L.M., D.DB., L. Ma..; methodology, E.DA., R.P., R.L., formal analysis, A.L., E.DA., L.G., MP., R.; resources, L.M.; data curation, D.DB., E. DA..; writingoriginal draft preparation, E.D.A., R.L.; writing-review and editing, L.M., D. DB.; supervision, L.M., D.DB, L.Ma..;

Funding: This research was funded by CNR ISPA.

Institutional Review Board Statement: The study was conducted in accordance with the Declaration of Helsinki, and ethical approval was obtained by the institutional review board (University Hospital of Bari, document number 33/2020);

Informed Consent Statement: Informed consent was obtained from all subjects involved in the study.

Acknowledgments: In this section, you can acknowledge any support given which is not covered by the author contribution or funding sections. This may include administrative and technical support, or donations in kind (e.g., materials used for experiments).

Conflicts of Interest: The authors declare no conflict of interest. The funders had no role in the design of the study; in the collection, analyses, or interpretation of data; in the writing of the manuscript, or in the decision to publish the results.

\section{References}

1. Burney, P.G.J.; Potts, J.; Kummeling, I.; Mills, E.N.C.; Clausen, M.; Dubakiene, R.; Barreales, L.; Fernandez-Perez, C.; FernandezRivas, M.; Le, T.M.; et al. The prevalence and distribution of food sensitization in European adults. Allergy Eur. J. Allergy Clin. Immunol. 2014, 69, 365-371, doi:10.1111/all.12341.

2. WHO/IUIS Allergen Nomenclature. Available online: www.allergen.org (accessed on 25 November 2021)

3. EU Regulation No. 1169/2011 of the European Parliament and of the Council of 25 October 2011 on the provision of food information to consumers. Official Journal of the European Union L 304, 18-63.

4. De Angelis, E.; Bavaro, S.L.; Forte, G.; Pilolli, R.; Monaci, L. Heat and pressure treatments on almond protein stability and change in immunoreactivity after simulated human digestion. Nutrients 2018, 10, 1-20, doi:10.3390/nu10111679.

5. Lyons, S.A.; Knulst, A.C.; Burney, P.G.J.; Fernandez-Rivas, M.; Ballmer-Weber, B.K.; Barreales, L.; Bieli, C.; Clausen, M.; Dubakiene, R.; Fernandez-Perez, C.; et al. Predicting food allergy: The value of patient history reinforced. Allergy Eur. J. Allergy Clin. Immunol. 2021, 76, 1454-1462, doi:10.1111/all.14583. 
6. Bavaro, S.L.; Di Stasio, L.; Mamone, G.; De Angelis, E.; Nocerino, R.; Canani, R.B.; Logrieco, A.F.; Montemurro, N.; Monaci, L. Effect of thermal/pressure processing and simulated human digestion on the immunoreactivity of extractable peanut allergens. Food Res. Int. 2018, 109, 126-137, doi:10.1016/j.foodres.2018.04.021.

7. De Angelis, E.; Pilolli, R.; Bavaro, S.L.; Monaci, L. Insight into the gastro-duodenal digestion resistance of soybean proteins and potential implications for residual immunogenicity. Food Funct. 2017, 8, 1599-1610, doi:10.1039/c6fo01788f.

8. Bavaro, S.L.; De Angelis, E.; Barni, S.; Pilolli, R.; Mori, F.; Novembre, E.M.; Monaci, L. Modulation of milk allergenicity by baking milk in foods: A proteomic investigation. Nutrients 2019, 11, 1-15, doi:10.3390/nu11071536.

9. Corallino, M.; Nico, A.; Kourtis, G.; Caiaffa, M.F.; MacChia, L. Skin testing technique and precision in stinging insect allergy. J. Clin. Nurs. 2007, 16, 1256-1264, doi:10.1111/j.1365-2702.2007.01842.x.

10. Ekezie, C.F.G.; Cheng, J.H.; Sun, D.W. Effects of nonthermal food processing technologies on food allergens: A review of recent research advances. Trends Food Sci. Technol. 2018, 74, 12-25, doi:10.1016/j.tifs.2018.01.007.

11. Zhang, Y.; Zhang, J.; Sheng, W.; Wang, S.; Fu, T.J. Effects of heat and high-pressure treatments on the solubility and immunoreactivity of almond proteins. Food Chem. 2016, 199, 856-861, doi:10.1016/j.foodchem.2015.12.063.

12. Venkatachalam, M.; Teuber, S.S.; Roux, K.H.; Sathe, S.K. Effects of roasting, blanching, autoclaving, and microwave heating on antigenicity of almond (Prunus dulcis L.) proteins. J. Agric. Food Chem. 2002, 50, 3544-3548, doi:10.1021/jf020012z.

13. Rahaman, T.; Vasiljevic, T.; Ramchandran, L. Effect of processing on conformational changes of food proteins related to allergenicity. Trends Food Sci. Technol. 2016, 49, 24-34, doi:10.1016/j.tifs.2016.01.001.

14. López, E.; Cuadrado, C.; Burbano, C.; Jiménez, M.; Rodríguez, J.; Crespo, J.F. Effects of autoclaving and high pressure on allergenicity of hazelnut proteins. J. Clin. Bioinforma. 2012, 2, 12, doi:10.1186/2043-9113-2-12.

15. Breiteneder, H. Classifying food allergens. In Detecting Allergens in Food; Koppelman, S. J. and Hefle, S. L., Eds. Woodhead Publishing Ltd, UK, 2006, Issue 117, pp. 21-61.

16. Skamstrup Hansen K., Ballmer-Weber B K, Lüttkopf D, Skov P S, Wüthrich B, Bindslev-Jensen C, Vieths S, Poulsen L.K. Roasted hazelnuts--allergenic activity evaluated by double-blind, placebo-controlled food challenge. Allergy 2003, 58, 132-8, doi: 10.1034/j.1398-9995.2003.23959.x.

17. Fierro V., Marzano V., Monaci L., Vernocchi P., Minnini M., Valluzzi R., Levi Mortera S., Pilolli R., Dahdah L., Calandrelli V., Bracaglia G., Arasi S., Riccardi C., Fiocchi A., Putignani L. Threshold of reactivity and tolerance to precautionary allergen labelled biscuits of baked milk and egg-allergic children. Nutrients 2021; 13: 4540. doi.org/10.3390/nu13124540

18. Costa, J.; Mafra, I.; Carrapatoso, I.; Oliveira, M.B.P.P. Hazelnut allergens: Molecular characterization, detection, and clinical relevance. Crit. Rev. Food Sci. Nutr. 2016, 56, 2579-2605, doi:10.1080/10408398.2013.826173.

19. Cabanillas, B.; Maleki, S.J.; Rodríguez, J.; Cheng, H.; Teuber, S.S.; Wallowitz, M.L.; Muzquiz, M.; Pedrosa, M.M.; Linacero, R.; Burbano, C.; et al. Allergenic properties and differential response of walnut subjected to processing treatments. Food Chem. 2014, 157, 141-147, doi:10.1016/j.foodchem.2014.02.025.

20. Cabanillas, B.; Cuadrado, C.; Rodriguez, J.; Hart, J.; Burbano, C.; Crespo, J.F.; Novak, N. Potential changes in the allergenicity of three forms of peanut after thermal processing. Food Chem. 2015, 183, 18-25, doi:10.1016/j.foodchem.2015.03.023.

21. Cuadrado, C.; Sanchiz, A.; Vicente, F.; Ballesteros, I.; Linacero, R. Changes induced by pressure processing on immunoreactive proteins of tree nuts. Molecules 2020, 25, 1-11, doi:10.3390/molecules25040954. 